\title{
Renal Cell Carcinoma With Cardiac Metastases
}

\author{
Steven Li Fraine ${ }^{\mathrm{a}, \mathrm{c}}$, Diana Coman ${ }^{\mathrm{a}}$, Madeleine Durand ${ }^{\mathrm{b}}$, Mikhael Laskine ${ }^{\mathrm{b}}$
}

\begin{abstract}
The median survival of metastatic renal cell carcinoma (mRCC) is 5 months with a 1 -year survival rate of $29 \%$. Cardiac metastasis from $\mathrm{RCC}$ is a rare finding and there is scarce data available on treatment options. Recently, the combination of nivolumab and ipilimumab has been approved as a first-line treatment for advanced RCC in patients with a poor prognosis. Here we present a case of a 45 -year-old male who presented to the emergency room with cough, dyspnea, and fever. Chest $\mathrm{X}$-ray showed hilar lymphadenopathy and diffuse reticulonodular opacities, whereas a thoracic computed tomography (CT) scan revealed carcinomatosis lymphangitis, pleural carcinosis and multiple heterogenous zones on the cardiac wall. A transthoracic echocardiogram and a cardiac magnetic resonance imaging (MRI) revealed cardiac metastases. Subsequent imaging showed abundant distal metastases whereas a renal biopsy confirmed clear cell RCC making it a high-grade stage IV metastatic RCC. The patient was treated with the combination of nivolumab and ipilimumab. The unique feature about this case is that we have found a rare case of cardiac metastases that persists after a 3-month follow-up. Previously, there was only one case report of a patient with RCC and cardiac metastases who showed persistent response to nivolumab after 12 months. The key points from this case report are that a high index of suspicion is required for diagnosing cardiac metastases given that the signs and symptoms of metastatic cardiac involvement can be non-specific. Spread has been described as directly through the renal vein and vena cava or indirectly via the lymphatic system, which confers a worse prognosis. Furthermore, cardiac metastases can be mistaken for thrombi, endocarditis, or primary tumors, therefore echocardiograms can be limiting. Supplemental imaging with cardiac MRI or positron emission tomography/CT (PET/CT) is often needed for further characterization.
\end{abstract}

Keywords: Renal cell carcinoma; Cardiac metastases; Immunotherapy; Tyrosine kinase inhibitors

\section{Introduction}

Cardiac metastases occur especially with leukemia, lympho-

Manuscript submitted March 28, 2021, accepted May 12, 2021

Published online July 10, 2021

aDepartment of Medicine, University of Montreal, Montreal, QC, Canada ${ }^{\mathrm{b}}$ Division of General Internal Medicine, Department of Medicine, University of Montreal, Centre Hospitalier de l'Universite de Montreal, Montreal, QC, Canada ${ }^{c}$ Corresponding Author: Steven Li Fraine, Department of Medicine, University of Montreal, Montreal, QC, Canada. Email: steven.lifraine@gmail.com

doi: https://doi.org/10.14740/wjon1376 ma, malignant melanoma or lung, esophageal and breast cancers [1]. They are uncommonly seen in conjunction with renal cell carcinoma (RCC). A high index of suspicion is required for diagnosing cardiac metastases, since signs and symptoms of metastatic cardiac involvement can be non-specific. It is most often a post-mortem finding [2]. Also, cardiac metastases can be easily mistaken for thrombus, endocarditis, myxoma, sarcoma or other primary tumors, so cardiac echocardiograms are not always reliable. Supplementary imagery such as cardiac magnetic resonance imaging (MRI) or 18 F-fluorodeoxyglucose positron emission tomography/computed tomography (FDG PET/CT) is often needed to further characterize the lesions [3]. The unique features about this case are that we have found cardiac metastases when least expected and that we have witnessed their stability after a 3-month immunotherapy treatment.

\section{Case Report}

A 45-year-old male presented to the emergency room (ER) with cough, dyspnea, fever, and fatigue. He had no known medical history besides occasional non-intravenous drug use and smoking. Physical examination was positive for rightupper quadrant pain and dyspnea but was otherwise non-contributory. A series of blood tests and imaging were undertaken.

Chest X-ray showed hilar lymphadenopathy and diffuse reticulonodular opacities whereas a thoracic $\mathrm{CT}$ scan revealed carcinomatosis lymphangitis, pleural carcinosis and multiple heterogenous zones on the cardiac wall. A transthoracic echocardiogram showed a left ventricular mass that was suspicious for metastasis. PET/CT described metabolically active lesions in the left ventricular septum and the right ventricular cavitary. A cardiac MRI further characterized a solid $12 \times 12$ $\mathrm{mm}$ mass on the left ventricular septal wall and a solid $11 \times$ $15 \mathrm{~mm}$ endovascular mass at the right ventricular apex (Fig. 1). These lesions were highly suggestive of metastasis due to the degree of contrast enhancement that was similar to other metastatic sites. There was no valvular or vena cava involvement. The left ventricular ejection fraction and pulmonary artery pressures were both normal. Subsequent imaging showed abundant distal metastases with hepatic, cerebral, adrenal, cutaneous, subcutaneous, muscular, and bone involvement. A right renal trocar biopsy was performed with histological morphology and immunohistochemistry compatible with RCC. Nuclear grading was a minimum of $3 / 4$ making it a high grade stage IV metastatic RCC (mRCC).

The patient's fever was attributed to a paraneoplastic 


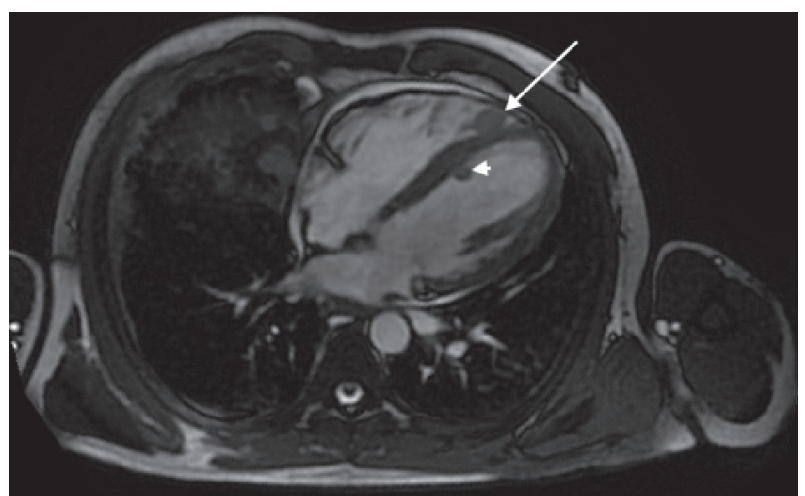

Figure 1. A cardiac magnetic resonance imaging showing a $12 \times 12$ $\mathrm{mm}$ left ventricular mass on the septal wall (arrowhead), and a $11 \times 15$ $\mathrm{mm}$ endovascular mass at the right ventricular apex (arrow).

phenomenon; a septic source was never been found. His right upper quadrant pain was attributed to hepatic capsule distention by the metastases. Blood tests revealed an inflammatory anemia (hemoglobin $(\mathrm{Hb}) 74 \mathrm{~g} / \mathrm{L}$ ), a reactive thrombocytosis triggered by his neoplasia, and hypercalcemia secondary to his bone metastases.

A bone marrow infiltration was not excluded but no bone marrow biopsy was performed during his stay. A genetics consult did not reveal any gene mutations predisposing to renal cancer.

With little evidence in the treatment of $\mathrm{mRCC}$ with cardiac involvement, the patient was started on nivolumab and ipilimumab due to their success in advanced mRCC. A combination of pembrolizumab with axitinib was initially considered but ultimately not chosen due to technical and social limitations along with the goal of beginning a treatment regimen as early as possible. The patient received two cycles of nivolumab/ipilimumab immunotherapy.

A scan 3 months later after two cycle of immunotherapy showed stability of the intracardiac masses. Unfortunately, the patient died soon after from a thrombotic stroke, likely in the context of thrombocytosis. An autopsy was not available to further study the pathological involvement.

\section{Discussion}

Here we present a case of cardiac metastases in the setting of $\mathrm{RCC}$. While a cardiac metastasis from RCC is a rare finding, its presence without vena cava involvement is extremely rare [4]. Spread has been described as directly through the renal vein and vena cava or indirectly via the lymphatic system. The latter confers a worse prognosis [5]. Since signs and symptoms of metastatic cardiac involvement can be non-specific, a high index of suspicion is required for diagnosis. It is frequently a post-mortem finding [2]. Also, cardiac metastases can be mistaken for thrombi, endocarditis, or primary tumors, therefore echocardiograms can be limiting. Supplemental imaging with cardiac MRI or PET/CT is often needed for further characterization [4]. In this case, the patient's symptoms were unlikely caused by the cardiac metastasis with no evidence of cardiac insufficiency, pulmonary hypertension, or valve disease. Diag- nosis was therefore dependent on investigating the ambiguous thoracic CT findings with advanced imaging.

The median survival of $\mathrm{mRCC}$ is 5 months with a 1 -year survival rate of $29 \%$ [6]. It is also very resistant to treatment whether it is radiotherapy, hormonotherapy, or cytotoxic agents [7]. The introduction of tyrosine kinase inhibitors (TKIs) has drastically changed the prognosis of patients with $\mathrm{mRCC}$. However, there is limited proof of efficacy for patients with heart metastases. For example, Schinzari et al showed a partial response with signs of metastatic devascularization with pazopanib [5]. Bazine et al showed stabilization of cardiac metastases with sunitinib [2]. Stellato et al showed partial regression of metastases with cabozantinib after a 3-month treatment period [8]. Besides the limited data available on treating heart metastases, TKIs can also present with toxic effects on the heart, which further precludes their use for this indication [9].

Recently, the combination of nivolumab and ipilimumab has been approved as a first-line treatment for advanced RCC in patients with poor prognosis [4]. Like TKIs, limited data are available on immune checkpoint inhibitors and their success in treating patients with RCC and cardiac metastases. To the best of our knowledge, Ansari et al reported the first case of a patient with RCC and intracardiac metastasis who showed persistent response to nivolumab after 12 months [4].

In summary, we highlight the complexity in the management of $\mathrm{mRCC}$ with cardiac involvement. In suspected patients, supplemental imaging should be completed to confirm cardiac metastasis so that a response to treatment may be monitored. Further studies can then assess the efficacity of treatment in such patients.

\section{Learning points}

Signs and symptoms of metastatic cardiac involvement can be non-specific; a high index of suspicion is required for diagnosis.

Metastatic spread to the heart has been described as directly through the renal vein and vena cava or indirectly via the lymphatic system, which confers a worse prognosis.

Cardiac metastases can be mistaken for thrombi, endocarditis, or primary tumors; therefore, echocardiograms can be limiting. Supplemental imaging with cardiac MRI or PET/CT is often needed for further characterization.

\section{Acknowledgments}

None to declare.

\section{Financial Disclosure}

None to declare.

\section{Conflict of Interest}

None to declare. 


\section{Informed Consent}

Informed consent was obtained from the patient. All patient information was de-identified.

\section{Author Contributions}

DC and SLF wrote the article and researched the supporting literature. ML and MD supervised the project and provided critical feedback. All authors reviewed and approved the final version of the manuscript.

\section{Data Availability}

The data supporting the findings of this study are available within the article.

\section{Abbreviations}

CT: computed tomography; MRI: magnetic resonance imaging; mRCC: metastatic renal cell carcinoma; PET: positron emission tomography; RCC: renal cell carcinoma; TKI: tyrosine kinase inhibitor

\section{References}

1. Zustovich F, Gottardo F, De Zorzi L, Cecchetto A, Dal Bianco M, Mauro E, Cartei G. Cardiac metastasis from renal cell carcinoma without inferior vena involvement: a review of the literature based on a case report. Two different patterns of spread? Int J Clin Oncol. 2008;13(3):271274.

2. Bazine A, Fetohi M, Tanz R, Mahfoud T, Ichou M, Errihani $\mathrm{H}$. Cardiac metastases of renal cell carcinoma revealed by syncope: diagnosis and treatment. Case Rep Oncol. 2014;7(2):560-564.

3. Chiles C, Woodard PK, Gutierrez FR, Link KM. Metastatic involvement of the heart and pericardium: CT and MR imaging. Radiographics. 2001;21(2):439-449.

4. Ansari J, Alhelali S, Albinmousa Z, Farrag A, Ali AM, Abdelgelil M, Alhamad A, et al. Rare case of intracardiac renal cell carcinoma metastasis with response to nivolumab: case report and literature review. Case Rep Oncol. 2018;11(3):861-870.

5. Schinzari G, Monterisi S, Signorelli D, Cona MS, Cassano A, Danza F, Barone C. Cardiac metastasis from renal cell carcinoma successfully treated with pazopanib: impact of TKIs' antiangiogenic activity. Tumori. 2014;100(6):e298300.

6. Reese AC, Whitson JM, Meng MV. Natural history of untreated renal cell carcinoma with venous tumor thrombus. Urol Oncol. 2013;31(7):1305-1309.

7. Canda AE, Kirkali Z. Current management of renal cell carcinoma and targeted therapy. Urol J. 2006;3(1):1-14.

8. Stellato M, Cursano MC, Citarella F, Pantano F, Russano M, Dell' Aquila E, Vincenzi B, et al. Response to cabozantinib in renal cell carcinoma with cardiac metastases. Anticancer Drugs. 2020;31(3):314-318.

9. Cheng H, Force T. Why do kinase inhibitors cause cardiotoxicity and what can be done about it? Prog Cardiovasc Dis. 2010;53(2):114-120. 